\title{
Pre-and Post-Transplant Serum Lactate Dehydrogenase Levels as a Predictive Marker for Patient Survival and Engraftment in Allogeneic Hematopoietic Stem Cell Transplant Recipients
}

\author{
Elham Roshandel ${ }^{1}$, Sayeh Parkhideh*1 ${ }^{*}$, Haniyeh Ghaffari Nazari ${ }^{1}$, \\ Mahshid Mehdizadeh ${ }^{1}$, Hossein Bonakchi ${ }^{1}$, \\ Ghazaleh Sankanian ${ }^{1}$, Abbas Hajifathali*1
}

\begin{abstract}
Background: The discovery of biomarkers to predict the development of complications associated with hematopoietic stem cell transplantation (HSCT) offers a potential avenue for the early identification and treatment of these life-threatening consequences. Serum lactate dehydrogenase (sLDH) has been identified as a potential biomarker for determining the outcome of allogenic HSCT (allo-HSCT).

Methods: A retrospective study was performed using data collected from 204 allo-HSCT recipient patients to examine the predictive value of sLDH levels pre- and post-allo-HSCT on patient survival, graft-versushost-disease (GVHD) incidence, and time to platelet/white blood cells (WBC) engraftment.

Results: Our findings show that neither pre- $(p=0.61)$ nor post-transplantation $(p=0.55)$ sLDH levels were associated with GVHD incidence. However, elevated SLDH levels pre- and post-transplantation $(\geq 386$ and $\geq 409 \mathrm{IU} / \mathrm{mL}$, respectively) were found to be adverse risk factors for patient survival $(p=0.16, p=0.20$, respectively). Furthermore, a median $\mathrm{SLDH}$ level $\geq 400 \mathrm{IU} / \mathrm{mL}$ from day +5 to day +15 post-transplantation had a significant positive association with enhanced time to platelet and white blood cell (WBC) engraftment, compared to patients with sLDH levels $<400 \mathrm{IU} / \mathrm{mL}$ ( $\mathrm{p}<0.001$ ).

Conclusions: Our data suggests that high sLDH levels pre- and post-allo-HSCT could be considered a predictor of poor patient survival. Furthermore, high levels of sLDH days 5-15 post-allo-HSCT could be associated with improved time to platelet and WBC engraftment; however, this appears to come at the cost of increased mortality risk.
\end{abstract}

Keywords: Engraftment, Graft versus host disease, Hematopoietic stem cell transplantation, Lactate dehydrogenase.

\section{Introduction}

Allogeneic hematopoietic stem cell transplantation (allo-HSCT) has been used as a curative treatment approach for a range of malignant and non-malignant diseases $(1,2)$. Despite the benefit this treatment strategy can provide, it is associated with potential life threatening complications These complications impact the transplantation outcome in these patients leading to morbidity and mortality. Recipient age, donor/recipient HLA compatibility, comorbidities, conditioning regimens, and graft-versus-host-disease (GVHD) prophylaxis are understood to be the main contributing factors for determining the risk of 
complications associated with allo-HSCT. However, these factors alone are unable to completely predict the success or failure of the allo-HSCT. Biomarkers offer a more effective alternative for determining the potential alloHSCT complications and outcomes (3).

Lactate dehydrogenase (LDH) is an enzyme found in all tissues of the body which catalyzing the reaction of pyruvate to lactate during glycolysis (3). Research has indicated that serum LDH (sLDH) levels are correlated with poor survival rate in patients with solid tumors and hematological malignancies. Given this relationship of sLDH with patient outcomes, the levels of sLDH may act as a valuable biomarker to predict the prognosis and survival of patients with various malignancies (4-6).

The predictive capacity of sLDH has also been observed in patients receiving HSCTs. Levels of sLDH have been shown to be elevated in patients following autologous HSCT (auto-HSCT) (7). A strong correlation between sLDH levels and stem cell mobilization has been reported in recipients of allo-HSCT and auto-HSCT (8). It has been observed that sLDH levels at the time of admission are an important predictive indicator for survival in patients with acute myeloid leukemia (AML) receiving HLAmatched sibling allo-HSCT (9). In patients with HLA-matched allo-HSCT, an elevated sLDH level, donor age $>45$, and poor performance status (2-3) appear to increase the risk for treatment-related mortality (TRM) (10). The study by Song et al. also has a similar result - aGVHD incidence is reduced with low level LDH in allogeneic sibling matched HSCTs (11) Despite this research, the value and role of sLDH level as a predictive indictor in determining transplantation-related complications and survival remains unclear.

The aim of this retrospective study was to examine the relationship of sLDH levels before and after allo-HSCT with the incidence of GVHD, survival, and engraftment time of platelet (PLT) and white blood cells (WBCs) in allo-HSCT recipients.

\section{Materials and Methods \\ Patients}

A total of 204 patients with malignant and nonmalignant hematological disorders who underwent allo-HSCT between 2009 and 2018 at Taleghani Hematopoietic Stem Cell Transplantation Center in Tehran, Iran, were included in this retrospective study. Patient clinical and laboratory data were collected from the clinical records. This study was approved by the ethical committee of Shahid Beheshti University of Medical Sciences, Tehran, Iran (IR.SBMU.REC.1398.148).

\section{Conditioning regimens}

The conditioning regimen for patients consisted of intravenous administration of Busulfan (0.8 $\mathrm{mg} / \mathrm{kg}$ every 6 hours for 4 days) followed by either Cyclophosphamide $(60 \mathrm{mg} / \mathrm{kg} /$ day for 2 days) or Fludarabine $\left(30 \mathrm{mg} / \mathrm{m}^{2}\right.$ of body surface area once a day for 5 days). The conditioning regimens were classified into distinct groups: Regimen 1 which consisted of Busulfan and Cyclophosphamide; Regimen 2 which included Busulfan and Fludarabine; and Regimen 3 which was comprised of a combination of Busulfan, Fludarabine, and Anti-Thymocyte Globulin (ATG). Depending on the patient condition, additional variations of the conditioning regiments were administered. In patients with Hodgkin's disease (HD) and nonHodgkin's lymphoma (NHL) a regimen 4 was prescribed which included Fludarabine (30 $\mathrm{mg} / \mathrm{m}^{2}$ for 5 days, IV), CCNU (1-2-chloroethyl3-cyclohexyl-1-nitrosourea) $\left(100 \mathrm{mg} / \mathrm{m}^{2}\right.$ for 2 days, oral), and Melphalan ( $40 \mathrm{mg} / \mathrm{m}^{2}$ for 1 day, IV). Regimen 5 was prescribed for patients with aplastic anemia (AA) and Fanconi anemia (FA) which consisted of Cyclophosphamide and ATG.

\section{GVHD prophylaxis}

All patients received GVHD prophylaxis which was composed of Cyclosporine A (CsA) and Methotrexate (MTX). A daily dose of 3 $\mathrm{mg} / \mathrm{kg} /$ day of CsA was intravenously (IV) from day -2 until +5 (the day of allo-HSCT is considered day zero) and $12.5 \mathrm{mg} / \mathrm{kg} /$ day by mouth (PO) until day +180 . Methotrexate 
(MTX) was administered IV from day +1 at a dose of $10 \mathrm{mg} / \mathrm{kg}$ and at days $+3,+6$ and +11 at a dose of $6 \mathrm{mg} / \mathrm{kg}$ in combination with CsA. In patient who received matched and mismatched unrelated donor allo-HSCT, $2.5 \mathrm{mg} / \mathrm{kg}$ of ATG for 2 days ( -1 and -2 ) was added to this combination.

\section{Hematopoietic stem cell transplantation}

Stem cells were sourced from peripheral blood mobilized via subcutaneous dministration of 5$10 \mu \mathrm{g} / \mathrm{kg} /$ day of granulocyte-colony stimulating factor (G-CSF) for 4-5 consecutive days. The enumeration of CD34+ donor peripheral blood cells was performed using flow cytometry (Attune NxT, Country) on day 5 post G-CSF administration using PE-conjugated human anti-CD34 (EXBIO, Czech Republic) to determine the optimal day for apheresis. Plasma reduction for $\mathrm{ABO}$ minor-mismatch and $\mathrm{RBC}$ depletion for major and bidirectional $\mathrm{ABO}$ mismatch grafts were performed on the apheresis product. RBC depletion was performed using hydroxyl ethyl starch (HES) 6\% (GRIFOLS, Spain). The number of CD34+ cells and CD3+ (FITC-conjugated, human, Beckman Coulter, Miami, FL, US) cells in the apheresis product were counted and viability tests on all apheresis yields were performed using Trypan Blue viability dye (Biowest, France) prior to transplantation.

\section{Determining the presence of $\mathrm{WBC}$ and platelet engraftment, GVHD, and supportive treatments}

WBC engraftment was defined as a peripheral WBC count $\geq 1.0 \times 10^{9} / \mathrm{L}$ for three consecutive days. Platelet engraftment was defined as the first of three consecutive days in which platelet count was $\geq 20 \times 10^{9} / \mathrm{L}$. The endpoints for the evaluation of WBC and PLT engraftment time were considered 30- and 50days post allo-HSCT, respectively. The presence of GVHD was diagnosed according to the National Institutes of Health (NIH) criteria (12). In the case of GVHD diagnosis, the first line treatment included dose adjusted CsA and methylprednisolone. Infection prophylaxis involved a combination of acyclovir (antiviral), ciprofloxacin (antibacterial), and fluconazole (antifungal). The threshold of hemoglobin levels was considered at or below $8 \mathrm{~g} / \mathrm{dL}$ for $\mathrm{RBC}$ transfusion. The PLT cut-off for platelet transfusion was $20 \times 109 / \mathrm{L}$. For blood transfusions, WBC depleted, and irradiated blood was used.

\section{Serum LDH measurement}

Serum LDH level was measured using the Hitachi 911 automatic chemistry analyzer (Roche) and a commercially available kit reagent. The sLDH concentration was reported as international units per liter (IU/L). Pre-transplant sLDH levels were defined as the median sLDH level from the day of admission to the day of HSCT (day zero). Post-transplantation sLDH levels were defined as the median sLDH level between day 0 to day +14 , median sLDH level at day +5 to day +15 , and the median sLDH level from day 0 to the last documented sLDH measurement.

\section{Statistical analyses}

Univariable binary logistic regression was used to investigate the relationship between sLDH and the incidence of acute GVHD. The end points of this study included overall survival, WBC and platelet engraftment. Overall survival was defined as the interval between the time of transplantation until the time of death from any cause (13). Univariable analysis of time to event was performed using the Cox proportional hazards model. Univariable and multivariable logistic regression was conducted to examine the association between risk factors and high sLDH levels in pre- and post-transplant patients. When performing the multivariable analysis, a backwards method with a significance level of $10 \%$ was used to select the features with the highest prognostic value. The calculations were carried out using SAS (version 9.4; SAS Institute Inc., Cary, NC, USA), with a significance level of $25 \%$ for the univariable logistic and Cox regressions. 


\section{Results}

\section{Patient characteristics}

Patient characteristics are shown in Table 1. The median level of sLDH on pre-HSCT from admission day to HSCT and post-HSCT at HSCT to day +14 , day +5 to +15 and
HSCT to last documented sLDH level record was 386, 409, 400, and 454 IU/L, respectively. Among the patients in this study, 161 (78.9\%) and $169(82.8 \%)$ had successful PLT and WBC engraftments, respectively.

Table 1. Patients' Characteristics.

\begin{tabular}{|c|c|c|c|}
\hline Characteristics & Median/ Frequency $(\%)$ & Characteristics & Median/Frequency (\%) \\
\hline Recipient Age & 32 & Donor Age & 32 \\
\hline Missing & $11(5.4 \%)$ & Missing & $68(33.3 \%)$ \\
\hline CD3 & 300 & $M N C$ & 6.4 \\
\hline Missing & $157(77 \%)$ & Missing & $17(8.3 \%)$ \\
\hline DP Gender & & Diagnosed disease & $13(6.40 \%)$ \\
\hline Male-Male & $56(27.5 \%)$ & NHL & $12(5.90 \%)$ \\
\hline Male-Female & $59(28.9 \%)$ & HD & $99(48.5 \%)$ \\
\hline Female-Female & $32(15.7 \%)$ & AML & $51(25.0 \%)$ \\
\hline Female-Male & $47(23 \%)$ & ALL & $7(3.40 \%)$ \\
\hline \multirow[t]{3}{*}{ Missing } & $10(14.9 \%)$ & Aplastic Anemia & $6(2.90 \%)$ \\
\hline & & Other* & $16(7.80 \%)$ \\
\hline & & Missing & \\
\hline Recipient BMI & $22(10.8 \%)$ & Donor BMI & $34(16.7 \%)$ \\
\hline Below 18.5 & $89(43.6 \%)$ & Below 18.5 & $69(33.8 \%)$ \\
\hline Between 18.5-24.9 & $57(27.9 \%)$ & Between 18.5-24.9 & $48(23.5 \%)$ \\
\hline Between 25-29.9 & $25(12.3 \%)$ & Between 25-29.9 & $32(15.7 \%)$ \\
\hline Above 30 & $11(5.4 \%)$ & Above 30 & $21(10.3 \%)$ \\
\hline Missing & & Missing & \\
\hline Recipient CMV antigen status & $19(9.3 \%)$ & Donor-recipient relationship & $145(71.1 \%)$ \\
\hline Negative & $144(70.6 \%)$ & Sibling & $43(21.1 \%)$ \\
\hline Positive & $41(20.1 \%)$ & Related & $16(7.8 \%)$ \\
\hline Missing & & Missing & \\
\hline Conditioning Regime & $103(50.5 \%)$ & GVHD prophylaxis & $123(60.3 \%)$ \\
\hline Regimen 1 & $46(22.5 \%)$ & CSA+MTX & $19(9.3 \%)$ \\
\hline Regimen 2 & $16(7.8 \%)$ & $\mathrm{CSA}+\mathrm{MTX}+\mathrm{ATG}$ & $62(30.4 \%)$ \\
\hline Regimen 3 & $4(2 \%)$ & Missing & \\
\hline Regimen 4 & $1(0.5 \%)$ & & \\
\hline Regimen 5 & $34(16.7)$ & & \\
\hline \multicolumn{4}{|l|}{ Missing } \\
\hline Blood Group & $59(28.9 \%)$ & Compatibility Blood Group & $107(52.5 \%)$ \\
\hline A & $41(20.1 \%)$ & Compatibility & $80(39.2 \%)$ \\
\hline $\mathrm{B}$ & $24(11.8 \%)$ & Incompatibility & $17(8.3 \%)$ \\
\hline $\mathrm{AB}$ & $62(30.4 \%)$ & Missing & \\
\hline $\mathrm{O}$ & $18(8.8 \%)$ & & \\
\hline \multicolumn{4}{|l|}{ Missing } \\
\hline GVHD Type & $59(76.6 \%)$ & Platelet engraftment & $161(78.9 \%)$ \\
\hline Acute & $18(23.4 \%)$ & Successful & $39(19.1 \%)$ \\
\hline \multirow{2}{*}{ Chronic } & & Failure & $4(2 \%)$ \\
\hline & & Missing & \\
\hline LDH pre_HSCT & 386 & WBC engraftment & $169(82.8 \%)$ \\
\hline Missing & $28(13.7 \%)$ & Successful & $31(15.2 \%)$ \\
\hline LDH post (Hsct to +14 Days) & 409 & Failure & $4(2 \%)$ \\
\hline Missing & $27(13.2 \%)$ & Missing & \\
\hline LDH post $(+5$ to +15 Days $)$ & 400 & & \\
\hline Missing & $30(14.7 \%)$ & & \\
\hline LDH post_HSCT & 454 & & \\
\hline Missing & $26(12.7 \%)$ & & \\
\hline
\end{tabular}

Demographic and clinical data of the allo-HSCT patients is demonstrated. Data are illustrated as Median and frequency (\%). SD, Standard deviation; DP, Donor-Patient; MNC, Mononuclear cell; NHL, non-Hodgkin's Lymphoma; HD, Hodgkin's Disease; ALL, Acute Lymphocytic Leukemia; AML, Acute Myeloid Leukemia; ALL Acute Lymphocytic Leukemia; AA, Aplastic Anemia; MAC, Myeloablative Conditioning Regimen; RIC, Reduced Intensity Conditioning; GVHD, Graft-versus-host disease; CysA, Cyclosporine A; MTX, Methotrexate; ATG, Anti-thymocyte globulin." Diagnoseddisease referred as “Other” comprised of Adrenoleukodystrophy (ALD), Myelodysplastic syndromes (MDS), and Thalassemia. 


\section{Association between sLDH levels and GVHD}

We conducted a univariable logistic regression to identify the effect of sLDH levels on the incidence of GVHD. As shown in Table 2, patients with sLDH levels over 386 IU/L pretransplant (admission day to HSCT) and sLDH levels over $409 \mathrm{IU} / \mathrm{L}$ post-transplant (day 0 to day +14 ) had $20 \%$ and $24 \%$ lower odds of GVHD, respectively. However, this relationship was not found to be statistically significant (75\% CI:(0.58-1.11); $\mathrm{p}=0.44) ;(75 \%$ CI:(0.60$1.17) ; \mathrm{p}=0.55)$.

Table 2. Association of SLDH with GVHD and OS

\begin{tabular}{|c|c|c|c|c|}
\hline \multicolumn{3}{|c|}{ GVHD } & \multicolumn{2}{|l|}{ OS } \\
\hline Variables & Odds Ratio (75\% CI) & $\mathbf{p}$ & HR (75\% CI) & $\mathbf{p}$ \\
\hline LDH Pre_Hsct & & 0.44 & & $0.16 *$ \\
\hline$\geq 386$ & $0.80(0.58-1.11)$ & 0.44 & $1.48(1.07-2.04)$ & 0.16 \\
\hline$<386\left(\mathrm{RL}^{1}\right)$ & - & - & - & - \\
\hline LDHPost_Hsct (Hsct to +14 Days) & & 0.35 & & $0.20^{*}$ \\
\hline$\geq 409$ & $0.76(0.55-1.06)$ & 0.35 & $1.44(1.03-2.01)$ & 0.20 \\
\hline$<409\left(\mathrm{RL}^{1}\right)$ & - & - & - & - \\
\hline & 1. Reference Level, * & ifican & & \\
\hline
\end{tabular}

\section{Association between SLDH and OS}

We also applied the Cox proportional hazards model to determine the role of sLDH levels on overall patient survival. The results of this analysis are presented in Table 2 . The patients with sLDH levels above $386 \mathrm{IU} / \mathrm{L}$ prior to transplantation had about a $48 \%$ increased risk of death, compared to patients with sLDH levels below 386 IU/L (75\% CI:(1.07-2.04); $p=$ $0.16)$. The patients with post-transplant sLDH levels higher than $409 \mathrm{IU} / \mathrm{L}$ had a $44 \%$ greater risk of death, in comparison with the patients with sLDH levels below 409 IU/L $(75 \%$ CI:(1.03-2.01); $\mathrm{p}=0.20)$.

\section{Association sLDH and engraftment PLT engraftment}

The relationship between sLDH levels with PLT engraftment was investigated. As presented in Table 3, a post-transplant sLDH level above 400 was positively associated with successful PLT engraftment, compared to patients with sLDH levels below 400 (HR: 1.79 ; $=75 \%$ CI:(1.47-2.18); $\mathrm{p}<0.001)$.

\section{WBC engraftment}

We evaluated the association of sLDH levels with WBC engraftment. The results in Table 3 show that post-transplant sLDH levels above 400 were strongly associated with successful engraftment, compared with sLDH levels below 400 (HR: 2.13; 75\% CI:(1.71-2.52); p< 0.001). From the multivariable analysis, post-transplant sLDH levels above 400 was significantly associated with improved WBC engraftment time, compared to patients with sLDH levels below 400 (HR: 2.03; 90\%CI:(1.52-2.70); p< $0.001)$.

Table 3. Association of sLDH with Platelet \& WBC Engraftments.

\begin{tabular}{lllll} 
& \multicolumn{2}{l}{ Platelet Engraftment } & \multicolumn{2}{c}{ WBC Engraftment } \\
\hline Variables & HR $(\mathbf{7 5 \%}$ CI $)$ & $\mathbf{p}$ & HR $(\mathbf{7 5 \%}$ CI $)$ & $\mathbf{p}$ \\
\hline LDH pre_Hsct & & 0.60 & & 0.83 \\
\hline$\geq 386$ & $0.91(0.75-1.11)$ & 0.60 & $0.96(0.80-1.16)$ & 0.83 \\
\hline$<386\left(\mathrm{RL}^{1}\right)$ & - & - & - & - \\
\hline LDH post_Hsct $(+5$ to + 15 Days) & & $\mathbf{0 < 0 . 0 0 1 *}$ & & $\mathbf{0 < 0 . 0 0 1 *}$ \\
\hline$\geq 400$ & $1.79(1.47-2.18)$ & $0<0.001$ & $2.13(1.75-2.58)$ & $0<0.001$ \\
\hline$<400\left(\mathrm{RL}^{1}\right)$ & - & - & - & - \\
\hline
\end{tabular}




\section{Association between risk factors and sLDH levels pre- and post-HSCT}

The relationship between risk factors and preand post-transplantation sLDH levels were examined and presented in Table 4 and Table 5, respectively. Male transplantation recipients had $40 \%$ reduced odds of having high sLDH levels compared to female recipients $(75 \%$ CI:(0.50-0.72); $\mathrm{p}=0.001)$. Among the different diagnoses, ALL and aplastic anemia appeared to have a significant effect on SLDH level. In patients with aplastic anemia, the odds of having elevated sLDH levels was $75 \%$ lower than patients with HD (75\% CI:(0.07-0.70); $\mathrm{p}=$ 0.15). However, patients with ALL had 2.62 times greater odds of having high sLDH levels compared to HD (75\% CI:(1.68-4.16); $\mathrm{p}=0.01)$. The odds of having high sLDH levels in patients positive for the cytomegalovirus (CMV) antigen was 94\% greater than those negative for the CMV antigen (75\% CI:(1.372.74); $\mathrm{p}=0.02)$. In recipients whose blood group was type $\mathrm{B}$, the odds of having high sLDH levels was increased by $70 \%$ compared to recipients with an $\mathrm{AB}$ blood type (75\%CI:(1.22-2.37); $p=0.06)$. Conversely, when the recipient blood type was $\mathrm{O}$, the odds of having high sLDH levels was decreased by $30 \%$, compared to recipients with an $\mathrm{AB}$ blood type (75\% CI:(0.52-0.95); $\mathrm{p}=0.18)$.

Table 4. Association of Risk Factors with Odds High sLDH (Pre-HSCT).

\begin{tabular}{|c|c|c|c|c|}
\hline \multirow{2}{*}{ Variables } & \multicolumn{2}{|c|}{ Univariable } & \multirow{2}{*}{$\begin{array}{c}\text { Multivariable }^{2} \\
\text { Adjusted OR }(90 \% \text { CI })\end{array}$} & \multirow[b]{2}{*}{$\mathbf{p}$} \\
\hline & OR $(75 \% \mathrm{CI})$ & $\mathbf{p}$ & & \\
\hline Recipient Age & $0.98(0.97-1.005)$ & 0.45 & & \\
\hline Recipient Gender & & & & $0.005 * *$ \\
\hline Male & $0.60(0.50-0.72)$ & $0.001 *$ & $0.58(0.42-0.80)$ & 0.005 \\
\hline Female $\left(\mathrm{RL}^{1}\right)$ & - & - & - & - \\
\hline Diagnosed disease & & 0.01* & & $\mathbf{0 . 0 2} * *$ \\
\hline NHL & $0.82(0.40-1.68)$ & 0.75 & $4.22(0.64-27.59)$ & 0.20 \\
\hline AML & $0.70(0.47-1.04)$ & 0.29 & $0.49(0.24-1.01)$ & 0.10 \\
\hline ALL & $2.62(1.68-4.16)$ & 0.01 & $2.03(0.88-4.65)$ & 0.15 \\
\hline Aplastic Anemia & $0.25(0.07-0.70)$ & 0.15 & $0.24(0.04-1.50)$ & 0.20 \\
\hline Other & $2.06(0.88-5.27)$ & 0.34 & $1.55(0.25-9.54)$ & 0.69 \\
\hline $\mathrm{HD}\left(\mathrm{RL}^{1}\right)$ & - & - & - & - \\
\hline Recipient CMV PCR & & & & $0.04 * *$ \\
\hline Positive & $1.94(1.37-2.74)$ & $\mathbf{0 . 0 2} *$ & $2.02(1.14-3.58)$ & 0.04 \\
\hline \multicolumn{5}{|l|}{ Negative $\left(\mathrm{RL}^{1}\right)$} \\
\hline Conditioning Regimen & & 0.91 & & \\
\hline Regimen 1 & $11.68(0->999)$ & 0.98 & & \\
\hline Regimen 2 & $10.40(0->999)$ & 0.98 & & \\
\hline Regimen 3 & $12.48(0->999)$ & 0.98 & & \\
\hline Regimen4 & $32.76(0->999)$ & 0.98 & & \\
\hline Regimen 5(RL $\left.{ }^{1}\right)$ & - & - & & \\
\hline GVHD Prophylaxis & & 0.56 & & \\
\hline $\mathrm{CSA}+\mathrm{MTX}+\mathrm{ATG}$ & $0.86(0.64-1.15)$ & 0.56 & & \\
\hline $\mathrm{CSA}+\mathrm{MTX}\left(\mathrm{RL}^{1}\right)$ & - & - & & \\
\hline Recipient Blood Group & & $0.23 *$ & & NS \\
\hline A & $0.95(0.70-1.28)$ & 0.85 & & \\
\hline $\mathrm{B}$ & $1.70(1.22-2.37)$ & 0.06 & & \\
\hline $\mathrm{O}$ & $0.70(0.52-0.95)$ & 0.18 & & \\
\hline $\mathrm{AB}\left(\mathrm{RL}^{1}\right)$ & - & - & & \\
\hline Recipient BMI & & 0.03* & & NS \\
\hline Below 18.5 & $0.47(0.29-0.75)$ & 0.06 & & \\
\hline Between 18.5-24.9 & $0.71(0.52-0.96)$ & 0.20 & & \\
\hline Between 25-29.9 & $0.69(0.49-0.97)$ & 0.21 & & \\
\hline Above $30\left(\mathrm{RL}^{1}\right)$ & - & - & & \\
\hline
\end{tabular}


Table 5. Association of Risk Factors with Odds High sLDH (Post-HSCT).

\begin{tabular}{|c|c|c|c|c|}
\hline \multirow[b]{2}{*}{ Variables } & \multicolumn{2}{|l|}{ Univariable } & \multirow{2}{*}{\begin{tabular}{|l|} 
Multivariable $^{2}$ \\
Adjusted OR $(90 \%$ CI) \\
\end{tabular}} & \multirow[b]{2}{*}{$\mathbf{p}$} \\
\hline & OR $(75 \% \mathrm{CI})$ & $\mathbf{p}$ & & \\
\hline Recipient Age & $1.01(0.99-1.03)$ & 0.42 & & \\
\hline Donor Age & $1.01(0.99-1.03)$ & 0.46 & & \\
\hline Patient Gender & & $0.05^{*}$ & & $0.04 * *$ \\
\hline Male & $0.75(0.62-0.89)$ & $0.05^{*}$ & $0.68(0.50-0.93)$ & 0.04 \\
\hline Female $\left(\mathrm{RL}^{1}\right)$ & - & - & - & - \\
\hline Donor Gender & & 0.256 & & \\
\hline Male & $0.83(0.70-1.002)$ & 0.256 & & \\
\hline Female $\left(\mathrm{RL}^{1}\right)$ & - & - & & \\
\hline Recipient BMI & & 0.01* & & NS \\
\hline Below 18.5 & $0.28(0.16-0.47)$ & 0.005 & & \\
\hline Between 18.5-24.9 & $1.02(0.75-1.38)$ & 0.93 & & \\
\hline Between 25-29.9 & $1.07(0.77-1.49)$ & 0.80 & & \\
\hline Above $30\left(\mathrm{RL}^{1}\right)$ & - & - & & \\
\hline Donor BMI & & $0.09 *$ & & NS \\
\hline Below 18.5 & $0.74(0.51-1.06)$ & 0.34 & & \\
\hline Between 18.5-24.9 & $0.66(0.49-0.87)$ & 0.09 & & \\
\hline Between 25-29.9 & $0.92(0.66-1.27)$ & 0.77 & & \\
\hline Above 30(RL $\left.{ }^{1}\right)$ & - & - & & \\
\hline DP Gender & & 0.13* & & NS \\
\hline Female-Female & $1.91(1.31-2.79)$ & 0.04 & & \\
\hline Female-Male & $0.82(0.70-1.12)$ & 0.47 & & \\
\hline Male-Male & $0.60(0.44-0.82)$ & 0.06 & & \\
\hline \multicolumn{5}{|l|}{ Male-Female $\left(\mathrm{RL}^{1}\right)$} \\
\hline Diagnosed disease & & 0.18* & & NS \\
\hline NHL & $2.00(0.95-4.21)$ & 0.28 & & \\
\hline AML & $0.80(0.54-1.18)$ & 0.51 & & \\
\hline ALL & $1.71(1.10-2.65)$ & 0.16 & & \\
\hline Aplastic Anemia & $1.50(0.59-3.77)$ & 0.60 & & \\
\hline Other & $0.20(0.06-0.59)$ & 0.08 & & \\
\hline $\mathrm{HD}\left(\mathrm{RL}^{1}\right)$ & - & - & & \\
\hline Recipient CMV PCR & & $0.07 *$ & & \\
\hline Positive & $1.65(1.19-2.28)$ & $0.07 *$ & & NS \\
\hline \multicolumn{5}{|l|}{ Negative $\left(\mathrm{RL}^{1}\right)$} \\
\hline Donor-recipient relationship & & 0.38 & & \\
\hline Related & $1.17(0.94-1.46)$ & 0.38 & & \\
\hline \multicolumn{5}{|l|}{ Sibling $\left(\mathrm{RL}^{1}\right)$} \\
\hline Conditioning Regimen & & 0.42 & & \\
\hline Regimen 1 & $0.004(0->999)$ & 0.97 & & \\
\hline Regimen 2 & $0.003(0->999)$ & 0.97 & & \\
\hline Regimen 3 & $0.012(0->999)$ & 0.97 & & \\
\hline Regimen4 & $>999(0->999)$ & 0.98 & & \\
\hline Regimen $5\left(\mathrm{RL}^{1}\right)$ & - & - & & \\
\hline GVHD Prophylaxis & & 0.26 & & \\
\hline CSA+MTX+ATG & $1.35(0.99-1.83)$ & 0.26 & & \\
\hline $\mathrm{CSA}+\mathrm{MTX}\left(\mathrm{RL}^{1}\right)$ & - & - & & \\
\hline ABO Compatibility & & 0.79 & & \\
\hline Match & $1.04(0.87-1.24)$ & 0.79 & & \\
\hline \multicolumn{5}{|l|}{ Mismatch $\left(\mathrm{RL}^{1}\right)$} \\
\hline Recipient Blood Group & & $0.04 *$ & & $0.02 * *$ \\
\hline A & $0.84(0.63-1.14)$ & 0.52 & $0.78(0.47-1.28)$ & 0.41 \\
\hline $\mathrm{B}$ & $1.98(1.40-2.79)$ & 0.02 & $2.79(1.57-5.20)$ & 0.00 \\
\hline $\mathrm{O}$ & $0.56(0.41-0.75)$ & 0.02 & $0.55(0.33-0.90)$ & 0.04 \\
\hline $\mathrm{AB}\left(\mathrm{RL}^{1}\right)$ & - & - & - & - \\
\hline Donor Blood Group & & 0.47 & & \\
\hline A & $1.20(0.85-1.67)$ & 0.53 & & \\
\hline $\mathrm{B}$ & $0.80(0.56-1.14)$ & 0.46 & & \\
\hline $\mathrm{O}$ & $0.69(0.49-1.02)$ & 0.26 & & \\
\hline $\mathrm{AB}\left(\mathrm{RL}^{1}\right)$ & - & - & & \\
\hline
\end{tabular}

1. Reference Level, 2. Backward Selection, *. Significant at 0.25, **. Significant at 0.10. 
In patients with a body mass index (BMI) below 18.5, the odds of having high sLDH levels was $53 \%$ lower than patients with a BMI above 30 (75\% CI:(0.29-0.75); $\mathrm{p}=0.06$ ). For patients with a BMI between 18.5-24.9 and 25-29.9, both groups had 30\% lower odds of having high sLDH levels compared to the highest level of BMI $\quad(75 \% \quad$ CI:(0.52-0.96); $\quad \mathrm{p}=0.20)$; (75\%CI:(0.49-0.97); $\mathrm{p}=0.21)$. Assuming the effects of all the other variables are constant, the results of the multivariable analysis indicate that the odds of having high sLDH levels in male patients was $42 \%$ lower compared to female patients (90\% CI:(0.42-0.80); $\mathrm{p}=0.005)$. Among the different diseases examined, patients with AML had 51\% lower odds of having high sLDH levels, compared to other diagnoses (90\%CI:(0.24-1.01); $\quad \mathrm{PP}=0.10$ ). Additionally, our findings indicate that patients positive for the CMV antigen had 2.02 times greater odds of having high sLDH levels compared to patients negative for the CMV antigen (90\%CI:(1.14$3.58) ; p=0.04$ ).

The results of our analysis for investigating the effects of risk factors on post-HSCT sLDH levels are shown in Table 5. Male recipients had a 25\% lower chance of having high sLDH levels post-HSCT compared to female recipients (75\% CI:(0.62-0.89); $\mathrm{p}=0.05)$. The patients with a BMI below 18.5 had $72 \%$ lower odds of having high sLDH levels $(75 \%$ CI:(0.16-0.47); $\mathrm{p}=0.005)$. In patients receiving from donors with a BMI between 18.5-24.9, recipients had $34 \%$ lower odds of having high sLDH levels (75\% CI:(0.49-0.87); $\mathrm{p}=0.09$ ). The female donor to female recipient combination had $91 \%$ higher odds of having high sLDH levels (75\% CI:(1.31-2.79); $\mathrm{p}=$ 0.04). The male donor to male recipient combination had $40 \%$ lower odds of having high sLDH levels (75\% CI:(0.44-0.88); $\mathrm{p}=$ 0.06). The patients with ALL had 1.71 times higher odds of having high sLDH compared to HD (75\% CI:(1.16-2.65); $\mathrm{p}=0.16$ ) and patients classified as "other" had $80 \%$ lower odds of having high sLDH levels (75\% CI:(0.06-0.59); $\mathrm{p}=0.08)$. The odds of having high sLDH levels in patients positive for the CMV antigen were $65 \%$ higher than patients negative for the CMV antigen (75\% CI:(1.19-2.28); $\mathrm{p}=0.07)$. The odds of having high sLDH levels was increased by $98 \%$ in patients with a B blood type $(75 \%$ CI:(1.40-2.79); $\mathrm{p}=0.02)$; however, patients with O blood type were found to have decreased odds of having high sLDH levels by $44 \%$ (75\% CI:(0.41-0.75); $\mathrm{p}=0.02)$. In the multivariable analysis, we can see that being male lowered the odds of having high sLDH levels by $32 \%$ (90\%CI:(0.50-0.93); $\mathrm{p}=0.04)$. The results of multivariable analysis were the same as univariable analysis for the recipient blood groups $\mathrm{B}$ and $\mathrm{O}$. Controlling for the confounding effects of other variables, blood group B in recipients had increased odds of having high sLDH levels up to 2.75 times whereas blood group $\mathrm{O}$ lowered the odds of having high sLDH levels by $45 \% \quad(90 \%$ CI:(1.57-5.20); $\mathrm{p}=0.00)$; (90\%CI:(0.33-0.90); $\mathrm{p}=0.04)$. The male patients had $32 \%$ lower odds of having high sLDH levels compared to females (90\%CI:(0.50-0.93); $\mathrm{p}=0.04)$.

The distribution frequency between outcomes and SLDH levels at different periods of time is presented in Table 6.

Table 6. Crosstab of Outcomes and sLDH at Different Periods of Time.

\begin{tabular}{|c|c|c|c|c|c|c|c|c|}
\hline \multirow[t]{2}{*}{$\begin{array}{c}\text { Outcomes } \\
\text { Periods }\end{array}$} & \multicolumn{2}{|c|}{$\begin{array}{l}\text { GVHD } \\
\text { N }(\%)\end{array}$} & \multicolumn{2}{|c|}{$\begin{array}{c}\text { Mortality } \\
\mathbf{N}(\%)\end{array}$} & \multicolumn{2}{|c|}{$\begin{array}{c}\text { WBC Engraftment } \\
\text { N (\%) }\end{array}$} & \multicolumn{2}{|c|}{$\begin{array}{c}\text { Platelet } \\
\text { Engraftment } \\
\text { N }(\%)\end{array}$} \\
\hline & Acute & Chronic & Death & Alive & Yes & No & Yes & No \\
\hline $\begin{array}{l}\text { Pre_HSCT } \\
\geq 386<386\end{array}$ & $\begin{array}{l}25(71.4 \%) \\
27(79.4 \%) \\
\end{array}$ & $\begin{array}{l}10(28.6 \%) \\
7(20.6 \%) \\
\end{array}$ & $\begin{array}{l}30(42.9 \%) \\
23(32.4 \%)\end{array}$ & $\begin{array}{r}40(57.1 \%) \\
48(67.6 \%) \\
\end{array}$ & $\begin{array}{l}74(84.1 \%) \\
76(86.4 \%) \\
\end{array}$ & $\begin{array}{l}14(15.9 \%) \\
12(13.6 \%)\end{array}$ & $\begin{array}{l}71(80.7 \%) \\
73(83.0 \%) \\
\end{array}$ & $\begin{array}{l}17(19.3 \%) \\
15(17.0 \%)\end{array}$ \\
\hline 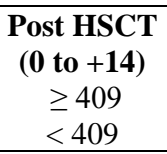 & $\begin{array}{l}27(71.1 \%) \\
26(81.3 \%)\end{array}$ & $\begin{array}{l}11(28.9 \%) \\
6(18.9 \%)\end{array}$ & $\begin{array}{l}28(40.6 \%) \\
26(35.6 \%)\end{array}$ & $\begin{array}{l}41(59.4 \%) \\
47(64.4 \%)\end{array}$ & $\begin{array}{l}- \\
-\end{array}$ & $\begin{array}{l}- \\
- \\
\end{array}$ & $\begin{array}{l}- \\
- \\
\end{array}$ & $\begin{array}{l}- \\
- \\
\end{array}$ \\
\hline $\begin{array}{c}\text { Post HSCT } \\
(+\mathbf{5} \text { to }+\mathbf{1 5}) \\
\geq 400 \\
<400 \\
\end{array}$ & - & - & $\begin{array}{l}- \\
-\end{array}$ & - & $\begin{array}{l}82(94.3 \%) \\
68(78.2 \%)\end{array}$ & $\begin{array}{c}8(5.7 \%) \\
19(21.8 \%)\end{array}$ & $\begin{array}{l}79(90.8 \%) \\
64(73.6 \%)\end{array}$ & $\begin{array}{c}8(9.2 \%) \\
23(26.4 \%) \\
\end{array}$ \\
\hline
\end{tabular}




\section{Discussion}

The use of sLDH as a predictive biomarker presents few barriers as measuring for it requires the use of an inexpensive and routine blood test. Previous research has evaluated the prognostic potential of sLDH levels in different diseases highlighting its utility as a biomarker (14-18). In the present study, we further explored the prognostic value of sLDH levels in the context of HSCT. Specifically, we examined the association of sLDH levels preand post-transplantation on its ability to determine overall survival, GVHD incidence, and PLT/WBC engraftment in patients receiving allo-HSCT. Furthermore, we evaluated the relationship between risk factors for HSCT complications and patient sLDH levels pre- and post-transplantation.

Our findings show that pre-transplantation, there is no significant relationship between levels of sLDH and GVHD development. This suggests that these levels have no predictive power in determining the incidence of GVHD pre-transplantation. Our data was consistent with the findings from Sivgin et al., in which no statistically significant relationship was found between the frequency of GVHD in patients and high levels of sLDH (19). The primary aim of our research was to evaluate the predictive value of post-transplant sLDH levels as an early biomarker for the risk of GVHD development. Therefore, we examined the association between the level of sLDH and the incidence of GVHD during the two-week period following transplantation. Previous research by Sung et al. has found lower sLDH levels to be strongly correlated to a lower incidence of GVHD ( $p=$ 0.023 ) (12). However, the findings of their study showed that SLDH levels within the first two weeks post-transplantation were not correlated with GVHD development, which corroborates the results of our work.

In exploring the effect of sLDH on survival rate, our findings revealed a statistically significant relationship between high sLDH levels in the pre-transplantation stage and poor survival rates in recipients (hazard ratio:1.48, CI: 1.07-2.04, $p=0.16$ ). Research by Shouval et al. suggest $\mathrm{LDH}$ is a prognostic indicator for allo-HSCT outcomes in patients with multiple myeloma (MM). Their findings showed that the 5 -years OS rate was $22 \%$ in patients with normal LDH levels, whereas those with LDH levels above the upper limit of normal had a 5\% OS rate (20). Research by Sivgin et al. observed an increased risk of mortality in patients with high levels of sLDH. However, contrary to our results, they did not find any significant relationship between high sLDH levels and a decrease in OS rate (hazard ratio $=1.31, \mathrm{CI}$ : $0.80-2.13, \mathrm{p}=0.286$ ). Their findings showed a significant association between high sLDH levels pre-transplant and decreased disease-free survival (DFS) rates in patients receiving alloHSCT. Therefore, their findings suggest that high sLDH levels prior to allo-HSCT may better reflect the potential for disease relapse which predicts DFS, compared to OS (19). We further examined the relationship between the sLDH level and mortality rate in patients at days 0 to +14 post-transplantation. Our results demonstrated that $\mathrm{sLDH}$ levels $\geq 409 \mathrm{IU} / \mathrm{mL}$ significantly increase $(p=0.2)$ the risk of death by $44 \%$. The work by Kalaycio et al. corroborates our findings, in which they reported that high sLDH (>330U/L) from the time of admission to discharge was an adverse risk factor for overall survival in AML patients receiving allo-HSCT. In their study, 15 of the 16 patients with sLDH levels $>330 \mathrm{U} / \mathrm{L}$ died highlighting the detrimental effect of high sLDH on mortality rates post-HSCT). Several pathological processes have been associated with increased sLDH levels including, hemolysis, cellular necrosis, and elevated tissue turnover (21). Additionally, a high sLDH level has been suggested to be a potential marker of organ damage in light chain amyloidosis patients (22). Although we did not evaluate the association between sLDH levels and alloHSCT-related organ damage, it is possible that the elevated levels of sLDH observed within the first 14 days post allo-HSCT may reflect the onset of organ damage.

With respect to the relationship between 
sLDH levels and PLT/WBC engraftment, our findings indicate that there is no significant association between pre-allo-HSCT SLDH levels and engraftment outcomes. Our findings were reflected in a separate retrospective analysis of 156 patients receiving all-HSCT in which no significant correlation between pretransplant sLDH levels and time to engraftment of PLT and/or WBC was found (19). Conversely, Gergis et al. reported that pretransplant sLDH levels above $487 \mathrm{IU} / \mathrm{L}$ were negatively associated with PLT engraftment $(\mathrm{p}=$ 0.04 ) in patients with advanced myelofibrosis undergoing allo-HSCT (23). We believe that the inconsistencies described among studies regarding the effect of pre-transplant sLDH levels and engraftment may be a result of varying definitions of what constitutes PLT and WBC engraftment, population heterogeneity, disease diagnosis, and cut-off points for what is considered low or high sLDH.

In terms of the relationship between posttransplant sLDH levels and engraftment, our findings indicate that high sLDH levels ( $\geq 400 \mathrm{IU} / \mathrm{L}$ ) throughout the first 15 days after transplantation are positively correlated with faster PLT and WBC engraftment time. Research by Song et al. also reported a significant association between earlier time to engraftment and elevated sLDH levels post allo-HSCT. Furthermore, they showed that a high sLDH level $(\geq 470 \mathrm{U} / \mathrm{L})$ at the third week after transplantation accelerates PLT $(\mathrm{p}=0.025)$ and WBC $(\mathrm{p}=0.008)$ engraftment in comparison to patients with sLDH levels below $470 \mathrm{U} / \mathrm{L}$. The presence of sLDH isoenzymes have been reported in malignancy and inflammation. It has been suggested that SLDH inhibitors have the ability to alleviate the inflammatory effects of cytokines in colon carcinoma cell (24). Moreover, Haas et al. has indicated that acute inflammation activates post-transcriptional protein synthesis leading to maturation and cell cycle activation of stem-like megakaryocyte committed progenitors (SLMkPs) and other megakaryocyte progenitors. This process causes the rapid replenishment of PLT and prevents PLT depletion during inflammation (25). Given this understanding, we propose that throughout the first few days following transplantation the presence of elevated sLDH levels, as a marker of inflammation, is positively associated with earlier engraftment time.

Lastly, we wanted to determine the relationship between recipient risk factors with sLDH levels pre- and post-HSCT. Gender, ABO type, disease diagnosis, BMI, and positive CMV antigen were all found to be risk factors significantly associated with high SLDH levels pre- and post-transplantation. Previous research has shown elevated sLDH levels in patients with sepsis and in the presence of infections, such as CMV (26). In the current study, a positive CMV status pre- and posttransplantation were risk factors for high sLDH level in patients. Furthermore, patients positive for CMV with high sLDH levels had an increased risk of mortality. It is understood that CMV infection is a major cause of complications following HSCT that could contribute to organ damage (27). Consequently, organ damage leads to an increase in sLDH level which may decrease survival. This has the potential to explain why patients with high sLDH levels and a positive CMV status were at increased risk for mortality in our study.

Overall, our findings indicate that sLDH levels could not predict the incidence of GVHD in our patients. This suggests that developing a panel of markers to predict GVHD may be a more promising strategy than a single biomarker. Using the sLDH to develop panel markers may be helpful for the improvement of GVHD prediction. Additionally, we report that high sLDH levels during the first few days postHSCT significantly improved PLT/ WBC engraftment, although it adversely affected patient survival.

\section{Acknowledgements}

The authors would like to thank the staff of the Hematopoietic Stem Cell Research Center, Shahid Beheshti University of Medical Sciences for making this research possible. We would also like to thank the University of Medical Sciences for their cherished support. 


\section{References}

1. Gyurkocza B, Rezvani A, Storb RF. Allogeneic hematopoietic cell transplantation: the state of the art. Expert Rev Hematol. 2010;3(3):285-99.

2. Bazinet A, Popradi G. A general practitioner's guide to hematopoietic stem-cell transplantation. Curr Oncol. 2019;26(3):187-191.

3. Paczesny S. Biomarkers for posttransplantation outcomes. Blood. 2018;17;131(20):2193-2204.

4. Hirschhaeuser F, Sattler UG, Mueller-Klieser W. Lactate: a metabolic key player in cancer. Cancer Res. 2011;71(22):6921-5.

5. Wulaningsih $\mathrm{W}$, Holmberg L, Garmo $\mathrm{H}$, Malmstrom H, Lambe M, Hammar $\mathrm{N}$, et al. Serum lactate dehydrogenase and survival following cancer diagnosis. $\mathrm{Br} \mathrm{J}$ Cancer. 2015;113(9):1389-96.

6. Petrelli F, Cabiddu M, Coinu A, Borgonovo K, Ghilardi M, Lonati V, et al. Prognostic role of lactate dehydrogenase in solid tumors: a systematic review and meta-analysis of 76 studies. Acta Oncol. 2015;54(7):961-70.

7. Bolwell B, Pohlman B, Kalaycio M, Wise K, Goormastic M, Andresen S. LDH elevation after autologous stem cell transplantation. Bone Marrow Transplant. 1999;24(1):53-55.

8. Egan K, Singh V, Gidron A, Mehta J. Correlation between serum lactate ehydrogenase and stem cell mobilization. Bone Marrow Transplant. 2007;40(10):931-4.

9. Kalaycio M, Rybicki L, Pohlman B, Dean R, Sweetenham J, Andresen S, et al. Elevated lactate dehydrogenase is an adverse predictor of outcome in HLA-matched sibling bone marrow transplant for acute myelogenous leukemia. Bone Marrow Transplant. 2007;40(8):753-8.

10. Mehta J, Gordon LI, Tallman MS, Winter $\mathrm{JN}$, Evens AO, Frankfurt O, et al. Does younger donor age affect the outcome of reducedintensity allogeneic hematopoietic stem cell transplantation for hematologic malignancies beneficially? Bone Marrow Transplant. 2006;38(2):95-100.

11. Song MK, Chung JS, Seol YM, Kwon BR, Shin HJ, Choi YJ, et al. Influence of lactate dehydrogenase and cyclosporine a level on the incidence of acute graft-versus-host disease after allogeneic stem cell transplantation. J Korean Med Sci. 2009;24(4):555-60.

12. Sung AD, Chao NJ. Concise review: acute graft-versus-host disease: immunobiology, prevention, and treatment. Stem Cells Transl Med. 2013;2(1):25-32.

13. Ando T, Yamazaki E, Ogusa E, Ishii Y, Yamamoto W, Motohashi K, et al. Hashimoto C. Body mass index is a prognostic factor in adult patients with acute myeloid leukemia. Int $\mathbf{J}$ Hematol. 2017;105(5):623-630.

13. Zhou GQ, Tang LL, Mao YP, Chen L, Li WF, Sun Y, et al. Baseline serum lactate dehydrogenase levels for patients treated with intensity-modulated radiotherapy for nasopharyngeal carcinoma: a predictor of poor prognosis and subsequent liver metastasis. Int $\mathbf{J}$ Radiat Oncol Biol Phys. 2012:82(3):e359-65.

14. Gkotzamanidou M, Kastritis E, Gavriatopoulou MR, Nikitas N, Gika D, Mparmparousi D, et al. Increased serum lactate dehydrongenase should be included among the variables that define very-highrisk multiple myeloma. Clin Lymphoma Myeloma leuk. 2011;11(5):409-13.

15. Wimazal F, Sperr WR, Kundi M, Vales A, Fonatsch C, Thalhammer-Scherrer R, et al. Prognostic significance of serial determinations of lactate dehydrogenase (LDH) in the follow-up of patients with myelodysplastic syndromes. Ann Oncol. 2008;19(5):970-6.

16. Bolwell B, Kalaycio M, Sobecks R, Andresen S, McBee M, Kuczkowski L, et al. Autologous hematopoietic cell transplantation for non-Hodgkin's lymphoma: 100 month follow-up. Bone Marrow Transplant. 2002;29(8):673-9.

17. Armand P, Kim HT, Ho VT, Cutler CS, Koreth J, Antin JH, et al. Allogeneic transplantation with reduced-intensity conditioning for Hodgkin and non-Hodgkin lymphoma: importance of histology for outcome. Biol Blood Marrow Transplant. 2008;14(4):418-25.

18. Mohajertehran F, Ayatollahi H, Jafarian A H, Khazaeni K, Soukhtanloo M, Shakeri M, et al. Overexpression of Lactate Dehydrogenase in the Saliva and Tissues of Patients with Head and Neck Squamous Cell Carcinoma. Rep Biochem Mol Biol. 2019;7(2):142-149. 
19. Sivgin S, Ozenmis T, Kaynar L, Kurnaz F, Sivgin H. Predictive Value of Pretransplant Serum Lactate Dehydrogenase (LDH) Levels for Survival in Patients who have undergone Allogeneic Hematopoietic Stem Cell Transplantation (alloHSCT). J Stem Cell Res Ther. 2012;2(118). 20. Shouval R, Teper O, Fein JA, Danylesko I, Shem Tov N, Yerushalmi R, et al. LDH and renal function are prognostic factors for longterm outcomes of multiple myeloma patients undergoing allogeneic hematopoietic stem cell transplantation. Bone Marrow Transplant. 2020;55(9):1736-1743.

21. Barcellini W, Fattizzo B. Clinical applications of hemolytic markers in the differential diagnosis and management of hemolytic anemia. Dis Markers. 2015;2015:635670.

22. Muchtar E, Dispenzieri A, Lacy MQ, Buadi FK, Kapoor P, Hayman SR, et al. Elevation of serum lactate dehydrogenase in AL amyloidosis reflects tissue damage and is an adverse prognostic marker in patients not eligible for stem cell transplantation. $\mathrm{Br} \mathrm{J}$ Haematol. 2017;178(6):888-895.
23. Gergis U, Kuriakose E, Shore T, Mayer S, Mark T, Pearse R, et al. Allogeneic transplantation for patients with advanced myelofibrosis: splenomegaly and high serum $\mathrm{LDH}$ are adverse risk factors for successful engraftment. Clin Lymphoma Myeloma Leuk. 2016;16(5):297-303.

24. Manerba M, Di Ianni L, Govoni M, Roberti M, Recanatini M, Di Stefano G. Lactate dehydrogenase inhibitors can reverse inflammation induced changes in colon cancer cells. Eur J Pharm Sci. 2017;96:37-44.

25. Haas S, Hansson J, Klimmeck D, Loeffler D, Velten L, Uckelmann $\mathrm{H}$, et al. Inflammationinduced emergency megakaryopoiesis driven by hematopoietic stem cell-like megakaryocyte progenitors. Cell stem cell. 2015;17(4):422-34.

26. Medović R, Igrutinović Z, RadojevićMarjanović R, Marković S, Rašković Z, Simović $A$, et al. Clinical and laboratory differences between Epstein-Barr and cytomegalovirus infectious mononucleosis in children. Srp Arh Celok Lek. 2016;144(1-2):56-62.

27. Mori T, Kato J. Cytomegalovirus infection/disease after hematopoietic stem cell transplantation. Int J Hematol. 2010;91(4):588-95. 